
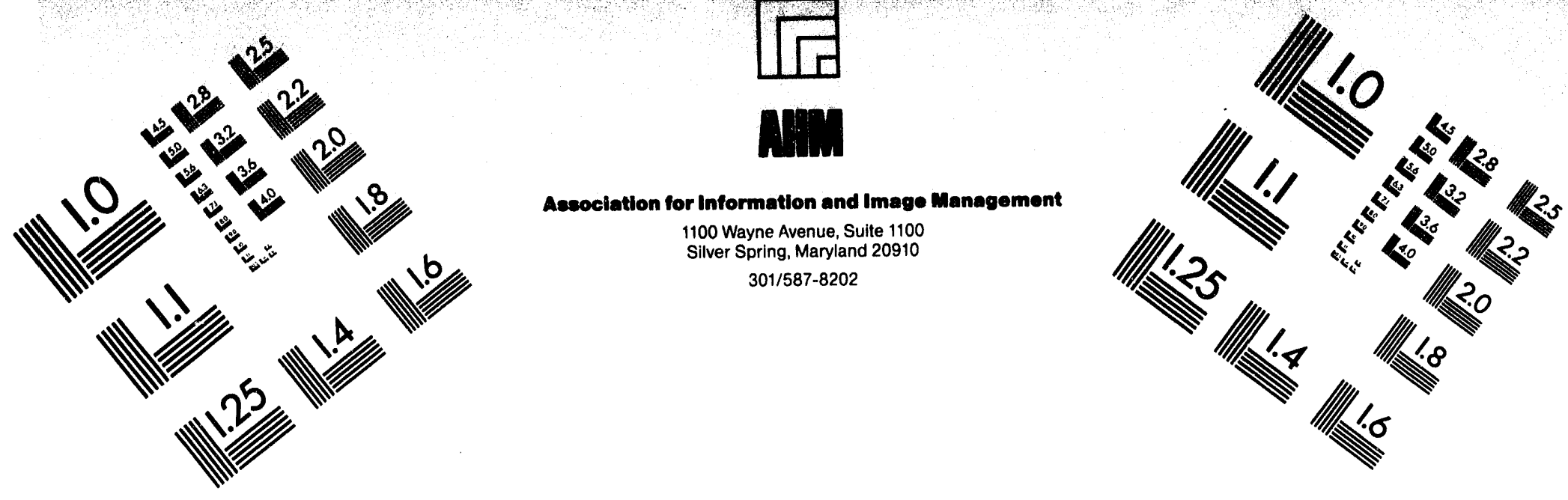

Centimeter

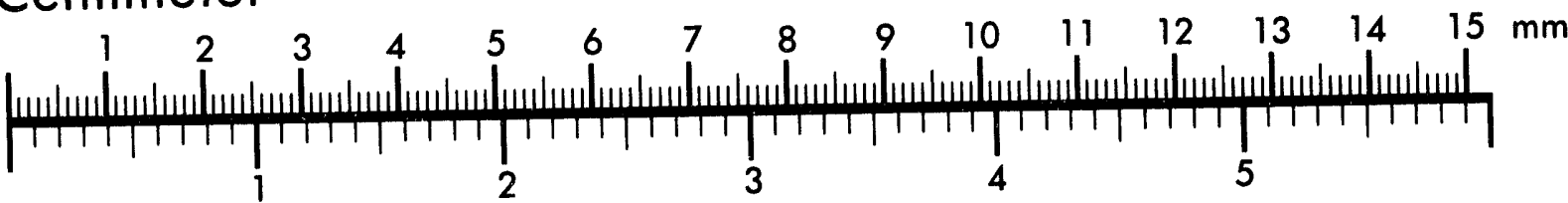

Inches

1100 Wayne Avenue, Sulte 1100
Silver Spring, Maryland 20910

301/587-8202
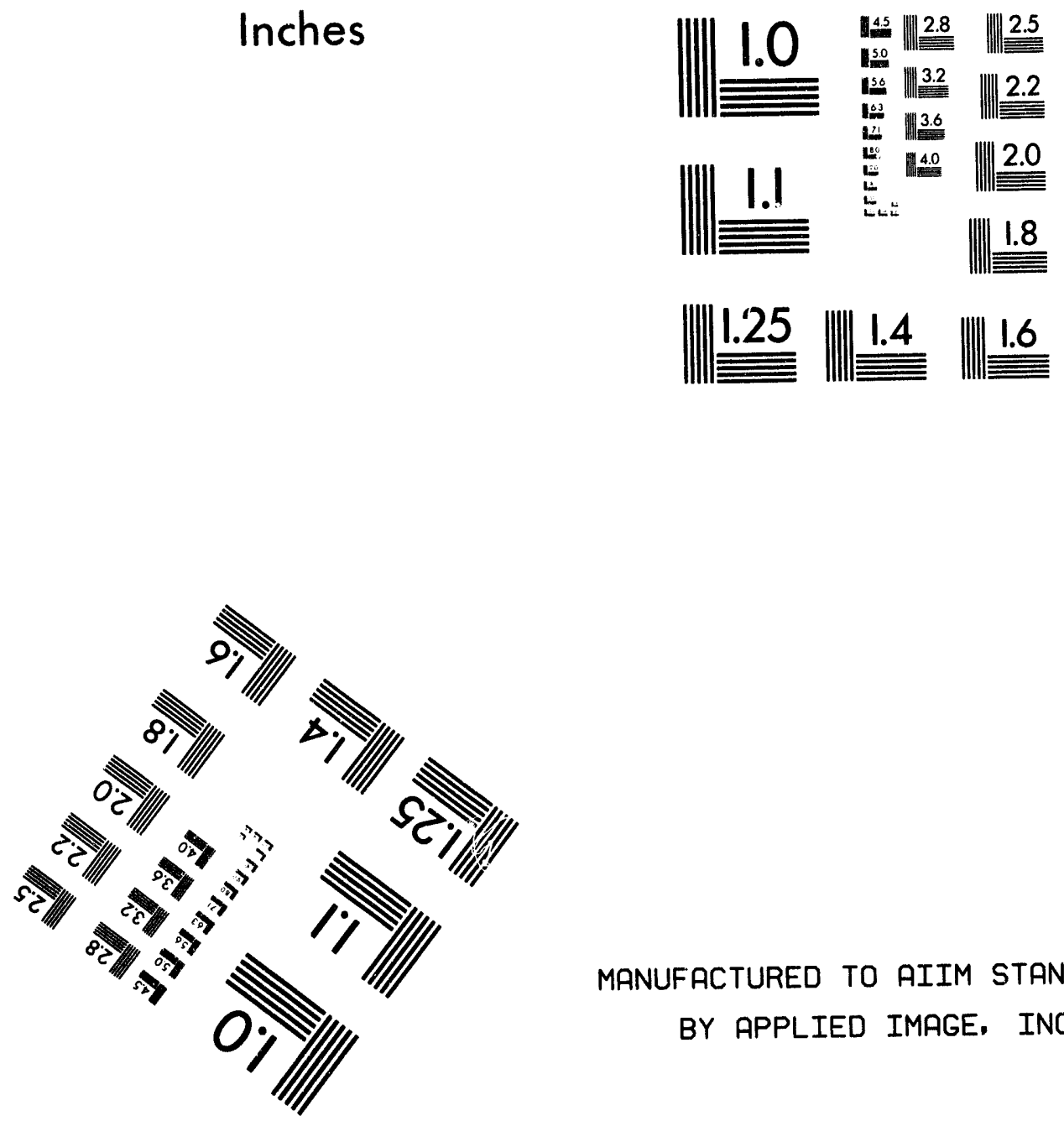

MANUFACTURED TO AIIM STANDARDS

BY APPLIED IMAGE. INC.

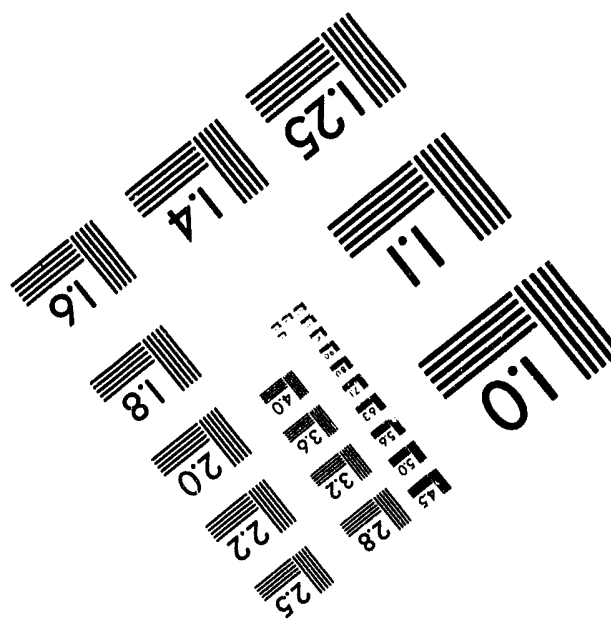



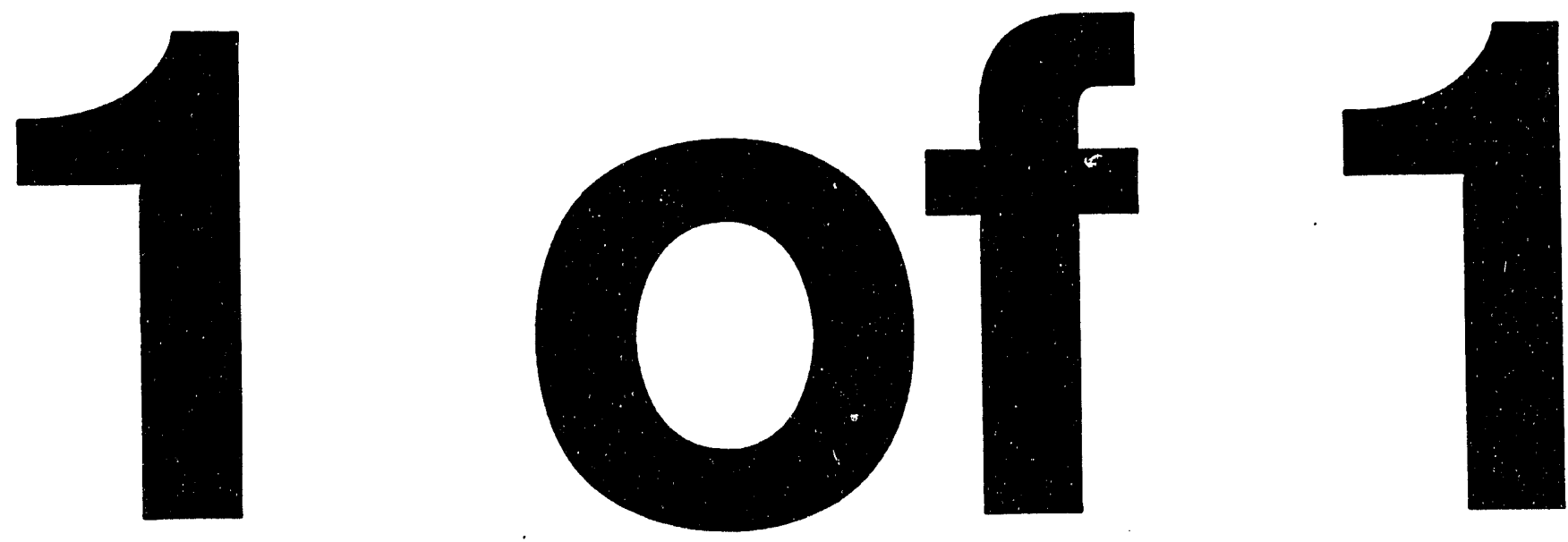


\title{
ULTRAFAST SCANNING PROBE MICROSCOPY
}

\author{
D. Botkin, S. Weiss, D.F. Ogletree, M. Salmeron, and D. S. Chemla
}

Department of Physics

University of California

Berkeley, CA 94720

\author{
Materials Sciences Division \\ Lawrence Berkeley Laboratory \\ University of California \\ Berkeley, CA 94720
}

January 1994

This work was supported by the Laboratory Directed Research and Development Program of Lawrence Berkeley Laboratory under the U.S. Department of Energy, Contract No. DE-AC03-76SF00098, and by ONR/DARPA urder Contract No. NO00-14.93.105.36.

\section{MASTER}




\title{
Ultrafast Scanning Probe Microscopy
}

\author{
D. Botkin, S. Weiss, D.F. Ogletree, M. Salmeron and D.S. Chemla \\ Department of Physics \\ University of California at Berkeley \\ and \\ Materials Sciences Division, MS 2-300 \\ Lawrence Berkeley Laboratory \\ University of California \\ Berkeley, CA 94720
}

\begin{abstract}
We have developed a general technique which combines the temporal resolution of ultrafast laser spectroscopy with the spatial resolution of scanned probe microscopy (SPM). Using this technique with scanning tunneling microscopy (STM), we have obtained simultaneous 2 ps time resolution and $50 \AA$ spatial resolution. This improves the time resolution currently attainable with STM by nine orders of magnitude. The potential of this powerful technique for studying ultrafast dynamical phenomera sn surfaces with atomic resolution is discussed.
\end{abstract}

\section{Introduction}

The development of mesoscopic systems and devices necessites the development of new experimental tools and techniques. Mesoscopic systems have typical dimensions much smaller than one micron and support dynamic excitations on timescales in the picosecond and sub-picosecond domain. Techniques exist which have either such spatial resolution or such temporal resolution. Ultrafast laser pulse spectroscopy can achieve femtosecond time resolution ${ }^{1}$, but the diffraction limit of optical beams limits spatial resolution to approximately one micron. The inventions of the Scanning Tunneling Microscope (STM) ${ }^{2}$ and the Atomic Force Microscope (AFM) ${ }^{3}$, which obtain atomic resolution, have revolutionized the field of surface science. However, acquisition electronics and scanning rates typically limit time resolution in these instruments to milliseconds. Therefore, to understand better the mechanisms governing mesoscopic systems and to optimize the devices that use them, it is necessary to develop new experimental techniques with simultaneous subpicosecondtemporal resolution and submicron-spatial resolution.

We proposed a general technique to wed ultrafast laser spectroscopy and SPM to obtain simultaneous picosecond time and atomic space resolution. 4 The STM, in particular, previously has been used as a detector in ultrafast measurements 5 , but the time resolution arose from nonlinear response in the sample; our technique adds ultrafast time resolution to the microscope itself. The basic concept is shown in Figure 1. Ultrafast time response occurs when the SPM probe has a nonlinear response to a short laser pulse. This nonlinearity can be essential to the nature of the probesample interaction or can be artificially introduced to the probe. For instance, the intrinsic

To be published in SPIE Proceedings (1994) 
nonlinearity of the STM I-V chara:teristic has been used to rectify microwaves and has recently been used to obtain ultrafast time resolution. ${ }^{7}$ By varying the relative time delay between the two pulses and integrating the resulting signal, a cross-correlation of the pump and prebe generated responses is obtained. The tip nonlinearity mixes the high frequency responses to produce a DC signal. Temporal resolution is defined by the temporal width of the nonlinear tip response and the width of the laser pulses. Spatial resolution should be comparable to the resolution of the normal SPM. At a fixed time delay, the tip can scan the surface, creating a snapshot of an ultrafast process. Several snapshots viewed in succession form a movie of surface dynamics. This basic principle is very general, and can be implemented for a wide variety of SPMs, based on tunneling, force, magnetic or electrostatic interactions. ${ }^{4}$ One example is the proposal to use an AFM lever as a microwave mixer to obtain time resolution. 8

\section{The Ultrafast Scanning Tunneling Microscope}

We have implemented this general technique in a specific device, the Ultrafast Scanning Tunnelng Microscope (USTM). The nonlinear element in this instrument is a fast photoconductive switch integrated with the STM tip assembly. Conceptually, the switch acts as a several picoseconds long gate. We used this instrument to image surfaces with 2 ps time resolution and better than $50 \AA$ spatial resolution.

A schematic of the experiment is shown in Fig. 2. A fast photoconductive switch, serially connected to the tip, provides the nonlinearity required for the correlation. The sample under study contains a high frequency coplanar transmission line and two side pads with photoconductive switches. One pad is

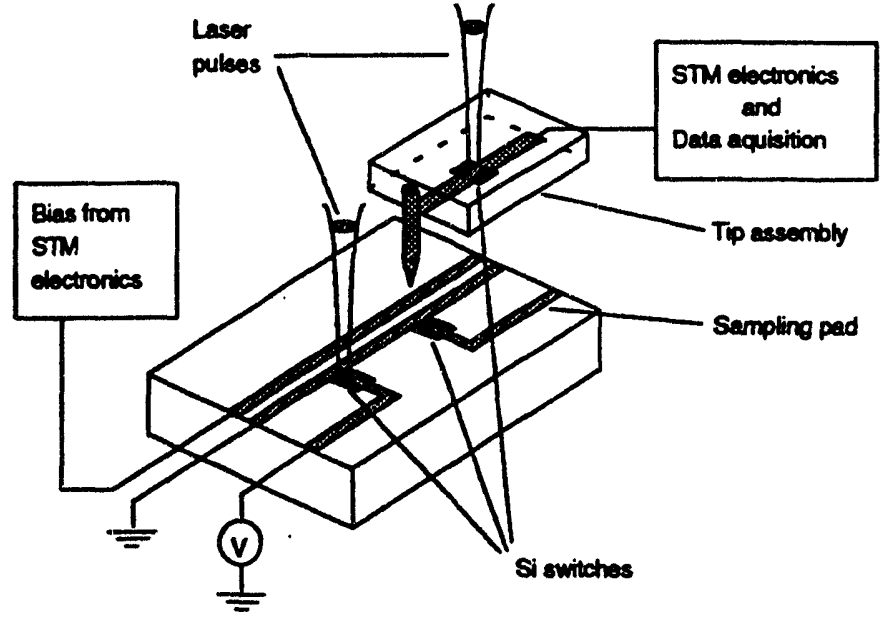

Figure 2. The USTM experiment. One laser pulse excites a voltage pulse on a transmission line. The second pulse photoconductively samples the tunneling current

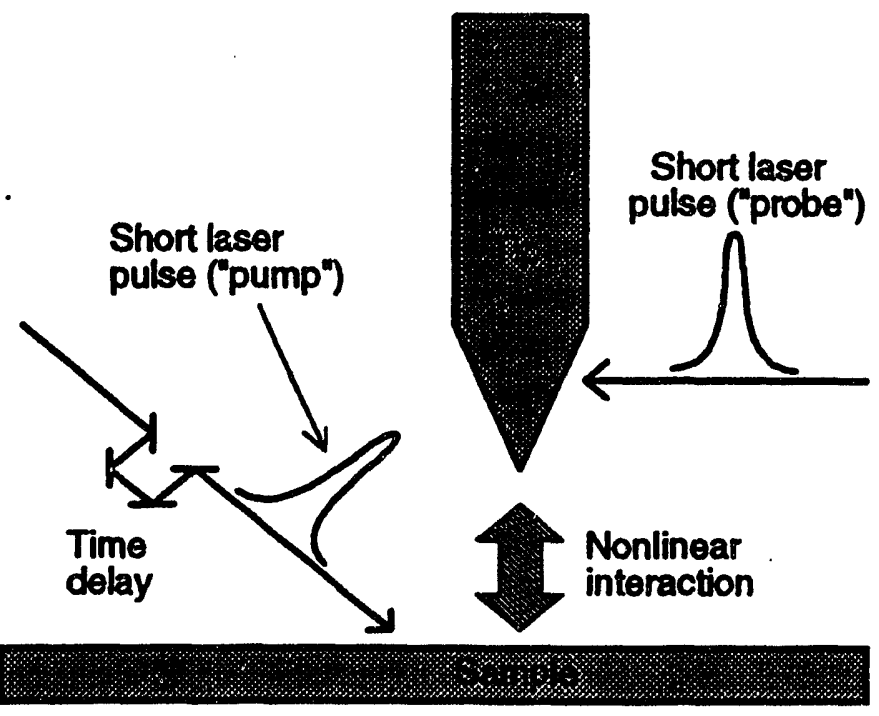

Figure 1. Ultrafast SPM (USPM) : Time resolution is achieved by modulating both the sample and the tip responses with two short optical pulses. A nonlinear tip respo.sse acts to correlate pump and probe signals. 
connected to a battery so that a laser pulse on the associated switch can generate a transient electrical pulse. The second pad is a sampling pad which is used to characterize the pulses propagating on the line. In a typical experiment, the first laser pulse excites an electrical transient on the transmission line. The second laser pulse samples the transient either on the STM tip or on the sampling pad.

The laser source was a $76 \mathrm{MHz}$ repetition rate modelocked Ti:Sapphire laser (Coherent Mira 900) which produced 120 fs pulses. The pulses were directed to the tip switch and to a sample switch through the same 10x long working distance objective. The spot size was $3-5 \mu \mathrm{m}$ in diameter and the average power in each beam was $25 \mathrm{~mW}$. The objective also formed part of an imaging system used to facilitate the tipsample approach.

Photographs of the STM instrument, the mounted sample and a close-up of the STM tip assembly appear in Fig. 3 a, b, and $c$, respectively. We designed and built a horizontally operating STM head based on a single piezo scanner tube. The sample was mounted on the scanner, and the tip assembly was fixed to the frame of the microscope. This design permitted a good optical access both to the sample and the tip. The STM was controlled by a commercial feedback electronics system. (RHK Technology, model STM 100).

The tip switch was fabricated from silicon on sapphire (SOS). ${ }^{9}$ It consisted of a single $\mathrm{Cr} / \mathrm{Au}$ line (60 $\mu \mathrm{m}$ wide) with a $3 \mu \mathrm{m}$ wide Si gap. It was illuminated through the sapphire substrate. The tip itself was fabricated from a brass wire $50 \mu \mathrm{m}$ in diameter, approximately $500 \mu \mathrm{m}$ long. We attached the tip to one end of the switch with a conductive silver epoxy. The other end of the switch was connected to the STM electronics through a current preamplifier. The "off" resistance of the switch was typically on the order of $30 \mathrm{M} \Omega$. This resistance was small enough to permit stable tunneling, provided that the gap resistance was at least comparable to the switch resistance. We were able to obtain atomic images of highly oriented pyrolytic graphite (HOPG) with the SOS tip assembly, without any light on the tip switch. The time averaged "on" resistance was on the order of $1 \mathrm{M} \Omega$, which suggests $100 \Omega$ resistance at the peak of the laser pulse.

The sample also was fabricated from SOS. The transmission line on the sample consisted of two $10 \mu \mathrm{m}$ wide $\mathrm{Cr} / \mathrm{Al} / \mathrm{Au}$ strips, separated by a $20 \mu \mathrm{m}$ gap. The generation and sampling switches had $5 \mu \mathrm{m}$ wide Silicon gaps. The generation pad was typically biased to $27 \mathrm{~V}$. Voltage transients on the transmission

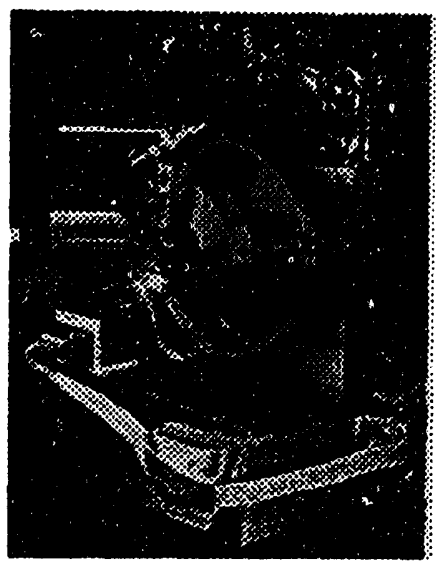

(a)

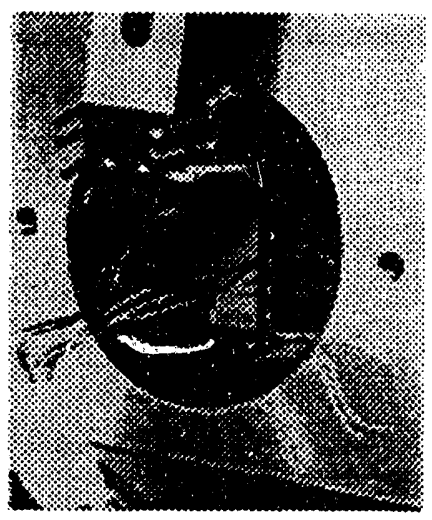

(b)

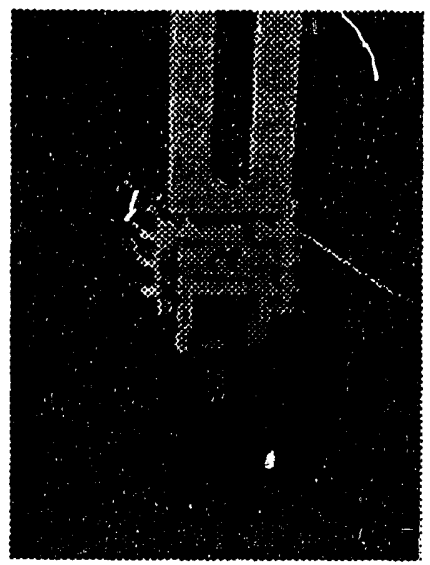

(c)

Figure 3. a) The STM base, b) the mounted tip and sample, c) detail of the tip assembly (The actual tip is too small to be visible) 
line were characterized with the aid of the sampling pad by conventional cross-correlation. 10 For a separation of $-100 \mu \mathrm{m}$ between the generation spot and the sampling spot, we routinely got $\sim 0.9-1$ ps FWHM cross-correlations which were unipolar and exhibited very little ringing. The actual pulse, a deconvolution of the measured signal, has a FWHM on the order of 600-700 fs. The tip-sample approach was done with both laser beams blocked. The STM tip was positioned over one strip of the transmission line 50 to $100 \mu \mathrm{m}$ away from the biased pad switch. This strip was biased by the STM electronics (typically 10 to 100 $\mathrm{mV}$ ). The second strip was connected to ground. Once in tunneling range, the sampling laser beam was directed to the tip assembly to gate the tunnel current.

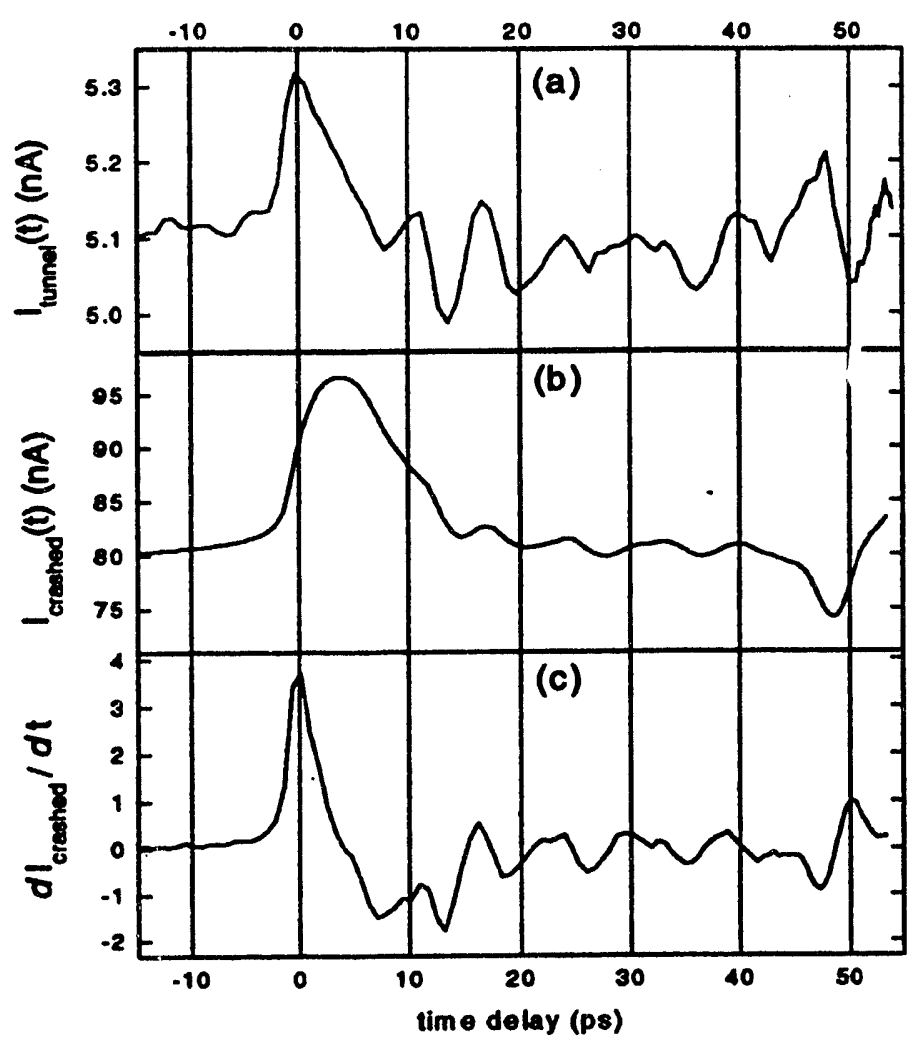

Figure 4. Time resolved current cross-correlation detected on the tip: (a) in tunneling range (5nA and $80 \mathrm{mV}$ settings), and (b) when the tip is crashed into the sample. (c) is the time derivative of (b).

\section{Results and Discussion}

A typical signal measured by the STM consists of a small AC component which varies with time delay riding on a large DC background. The background is simply the nominal tuneling current, given by (bias voltage)/(gap resistance). Fig 4 a shows a 4.5 ps wide cross-correlation pulse detected by the STM while tunneling at $5 \mathrm{nA}$ with a $+80 \mathrm{mV}$ bias on the strip. The $10 \%$ to $90 \%$ rise time is $2 \mathrm{ps}$. The average tunnel current is increased by $\sim 0.25 \mathrm{nA}$ when the transmission line pulse passes beneath the tip at zero time delay. This increase corresponds to a signal/background ratio, $\Delta \mathbf{I} /$, of $5 \%$. Fig. $4 \mathrm{~b}$ shows a cross-correlation recorded while the tip was in ohmic contact with the sample.

There are several features in this data. The large signal at a time delay of $47 \mathrm{ps}$ is a reflection from one end of the sample transmission line. This reflection also appeared in our standard sampling measurements. The structure following the main correlation peak is due to ringing in the tip structure, as we will show below. The FWHM of the correlation peak in Fig. $4 \mathrm{~b}$ is much broader ( $10 \mathrm{ps)}$ than the pulse on the transmission line. The broadening is, most likely, a result of dispersion of the electrical pulse on the tip. The bipolar correlation peak in Fig. $4 \mathrm{a}$ is significantly different from what we would expect for a purely ohmic response; in fact it bears some resemblance to Fig. $4 \mathrm{c}$, which is the numerical derivative of the trace in Fig. 4b. Apparently, a large portion of the tunneling signal comes from a capacitive-like response. However, the 
tunneling gap height dependence of this capacitive-like response is very different from the one expected for the geometrical capacitance of the junction; as we will show below, the geometrical capacitance of the tip assembly contributes negligibly to the signal. The existance of an intrinsic capacitance associated with the tunneling process has been discussed in the context of Coulomb Blockade (CB) and Single Electron Tunneling (SET). 11 This capacitance is beleived to be very small (on the order of $10^{-18} \mathrm{~F}$ ). The RC discharge time for such a capacitance at reasonable tunneling gap resistances is on the order of a few ps and can be directly investigated with our USTM.

Fig. 5a displays a series of crosscorrelation pulses recorded by the STM while tunneling with a $+80 \mathrm{mV}$ bias on the strip for gap resistances from $16 \mathrm{M} \Omega$ to 512 $\mathrm{M} \Omega$. For clarity, the DC background in each curve has been removed. In Fig. 5b, we normalize the peak height of the traces in Fig. 5a to unity in order to show changes in the lineshape of the signal. The figure shows that the fall time of the correlations increases with increasing gap resistance. This increase may be associated with the RC discharge time discussed above.

The spectra of the traces in Fig. 5a are : shown in Fig. 5c. These spectra were calculated for data in the range -10 ps to 37 ps. Additionally, the heights of the low frequency peaks have been normalized to 1 . The luw frequency peak describes the main bipolar correlation peak. The smaller high frequency peaks represent the ringing effect in the correlation traces. This ringing is probably due to an impedance mismatch between the tip and the sample and between the tip and the sampling switch. As the tunneling resistance is reduced, the mismatch is reduced, and the amplitude of the ringing peak is reduced. The fine structure in the ringing has not been identified.
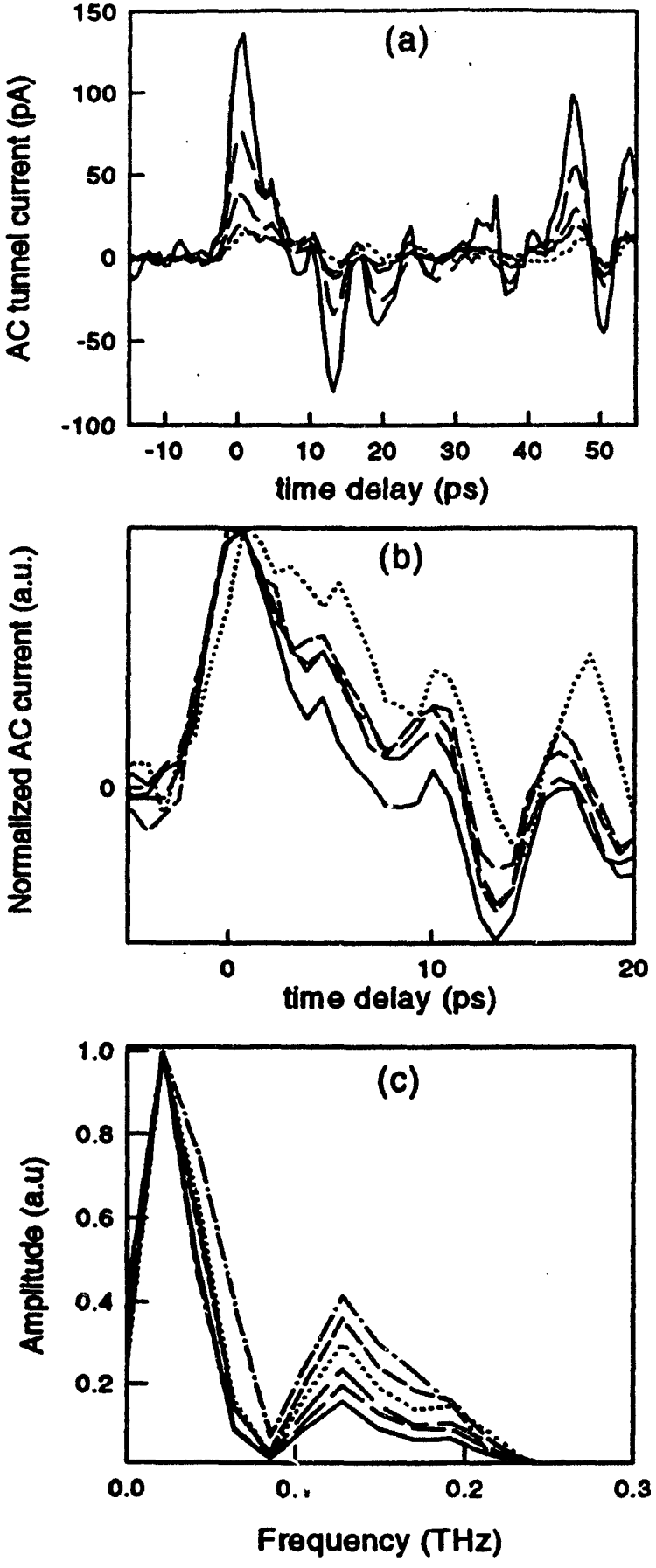

Figure 5. a) The tunnel-current $\Delta \mathrm{I}(\Delta t)$ for different gap resistances. (solid line is $16 \mathrm{M} \Omega$, long dash - $32 \mathrm{M} \Omega$, medium dash - $64 \mathrm{M} \Omega$, short dash - $128 \mathrm{M} \Omega$, dotted- $256 \mathrm{M} \Omega$, dash-dot - 512

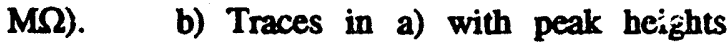
normalized to unity. c) Spectra of the curves in a). 
The height of the correlation peak in each trace, as function of the corresponding DC current is shown in Fig. 6. The filled squares represent measurements at 16, 32, 64, 128, 256 and 512 $\mathrm{M} \Omega$ gap resistances (the point at the top right of the figure corresponds to $16 \mathrm{M} \Omega$ ). The dashed line is a linear regression to the data. It is clear from the figure that the AC part of the tunneling current has the same gap impedance, and hence distance, dependence as that of the DC current. Moreover, when the tip is withdrawn from the surface by $50 \AA$, not only does the DC tunnel current vanish (as expected), but the AC part nearly vanishes as well. This means that the observed cross-correlation signal has little or no contribution from stray capacitance in the leads or from radiative coupling.

A detailed study of the nature and the origin of the tunnel-gap capacitance is currently under way. By varying the tip length, the gap conditions (by controlling the oxide layer) and the bias voltage on the transmission line, we will separate the contribution of the stray capacitance of the leads and reflections along the tip from the intrinsic response of the tunneling junction itself. We plan to improve the time resolution of our USTM by microfabricating the entire tip-switch assembly.

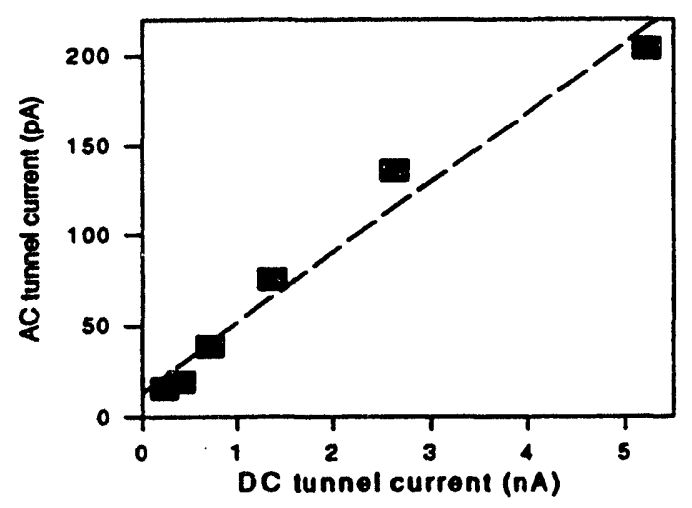

Figure 6. Amplitude of the time resolved signal versus $D C$ background. The dotted line is a linear regression to the data.

\section{Future Prospects}

One of the most exciting prospects for USPM is the potential to create movies of surface dynamics. Fig. 7 shows a $0.7 \times 0.7$ $\mu \mathrm{m}^{2}$ image of one of the transmission line conductors acquired as the pulse passed under the tip, at a time delay of 0 ps. The indentation at the center of the image was produced by crashing the tip into the strip. A similar image was acquired prior to the arrival of the pulse, at a time delay of -2.7 ps). We do not attribute features in the image to the propagation of the voltage pulse since the transmission line propagation velocity is $-200 \mu \mathrm{m} / \mathrm{ps}$, too fast to be resolved with this instrument.

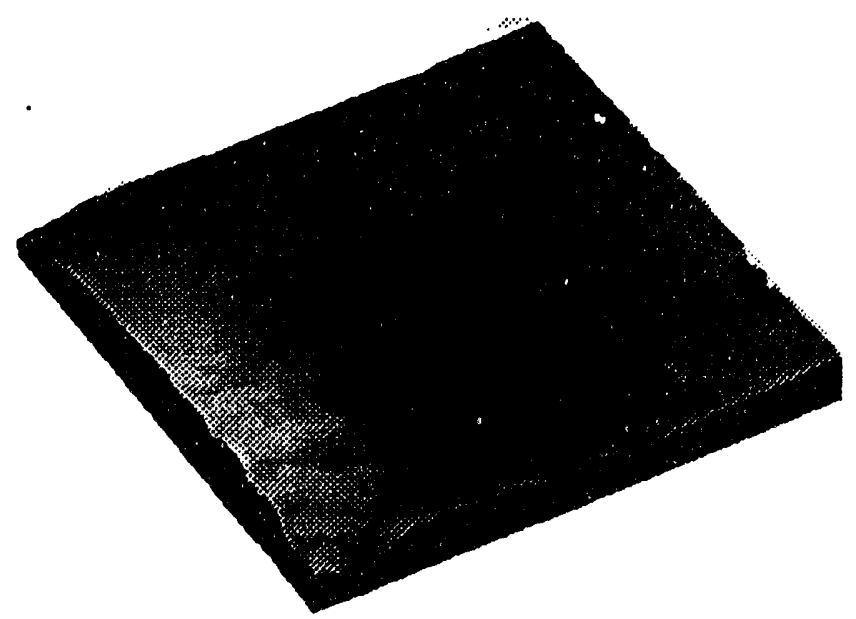

Figure 7. A topographic image $\left(0.7 \times 0.7 \mu \mathrm{m}^{2}\right)$ of the transmission line, taken with the exact same conditions as in Fig. 4a, at a time delay of 0 ps.

We have taken two frames of a movie; unfortunately, for this type of action, an electrical pulse propagating on metal, the camera is still too slow.

We believe that by improving the sensitivity, dynamic range, and time resolution of this 
technique, we will be able to animate surface dynamics by collecting a series of SPM images for increasing values of time delay. This will allow us to study dynamical phenomena in real space with atomic resolution. Such a tool will open a window for the observation of processes and excitations which propagate at velocities of a few $\AA$ per fs (or less). It should be possible to spatially resolve in real time phenomena such as vibronic motion on the atomic scale, carrier transport in semiconductor structures, electric field and voltage wavefront propagation at metal semiconductor interfaces. The operation of sub-micron electronic/optoelectronic devices can be directly characterized with such a technique.

USPM is not limited to the STM. As we discussed before, ${ }^{4}$ the non-linear nature of the probesample interactions in STM, AFM and near field optical microscopy (NSOM) offer many opportunities for resolving ultrafast phenomena on a nanometer scale.

\section{Acknowledgements}

The authors would like to thank J. Beeman for tremendous help with tip fabrication. This work was supported by the Laboratory Directed Research and Development Program of Lawrence Berkeley Laboratory under the U.S. Department of Energy, Contract No. DE-AC0376SF00098 and by ONR/DARPA under Contract No. N000-14.93.105.36.

\section{References}

IW. Kaiser. (ed.) Ultrashort Laser Pulses and Applications, Topics Appl. Phys. Vol. 60 (Springer, Berlin, Heidelberg 1988) p. 1.

2G. Binnig and H. Rohrer, Helvetica Physica Acta, 55, 726 (1982).

${ }^{3}$ G. Binnig, C.F. Quate and Ch. Gerber, Phys. Rev. Lett., 56, 930 (1986).

${ }^{4}$ S. Weiss, D. Botkin and D.S. Chemla, OSA proceedings on Ultrafast Electronics \& Optoelectronics, vol.14, San Francisco, January 25 1993, edited by J. Shah and U. Mishra, (OSA, Washington D.C. 1993), pp.162-165.

SR.J. Hamers and D.G. Cahill, Appl. Phys. Lett., 57, 2031 (1990) and J. Vac. Sci. Technol., B 9, 514 (1991).

${ }^{6}$ W. Krieger, T. Suzuki, M. VÖlcker and H. Walther, Phys. Rev. B 41, 10229 (1990) and references cited therin.

$7^{7}$ G. Nunes and M.R. Freeman, Science, 262, 1029 (1993).

${ }^{8}$ A.S. Hou, F. Ho and D.M. Bloom, OSA proceedings on Ultrafast Electronics \& Optoelectronics, vol.14, San Francisco, January 25 1993, edited by J. Shah and U. Mishra, (OSA., Washington D.C. 1993), pp.166-168.

${ }^{9}$ M.B. Ketchen, D. Grishkowsky, T.C. Chen, C-C. Chi, I.N. Duling, III, N.J. Halas, J-M. Halbout, J.A. Kash and G.P. Li, Appl. Phys. Lett. 48, 751 (1986).

10D.H. Auston IEEE J. Quantum Electron. QE-19, 639 (1983).

${ }^{11}$ For example, K.K Likharev, IBM J. Res. Develop., 32, 144 (1988). 

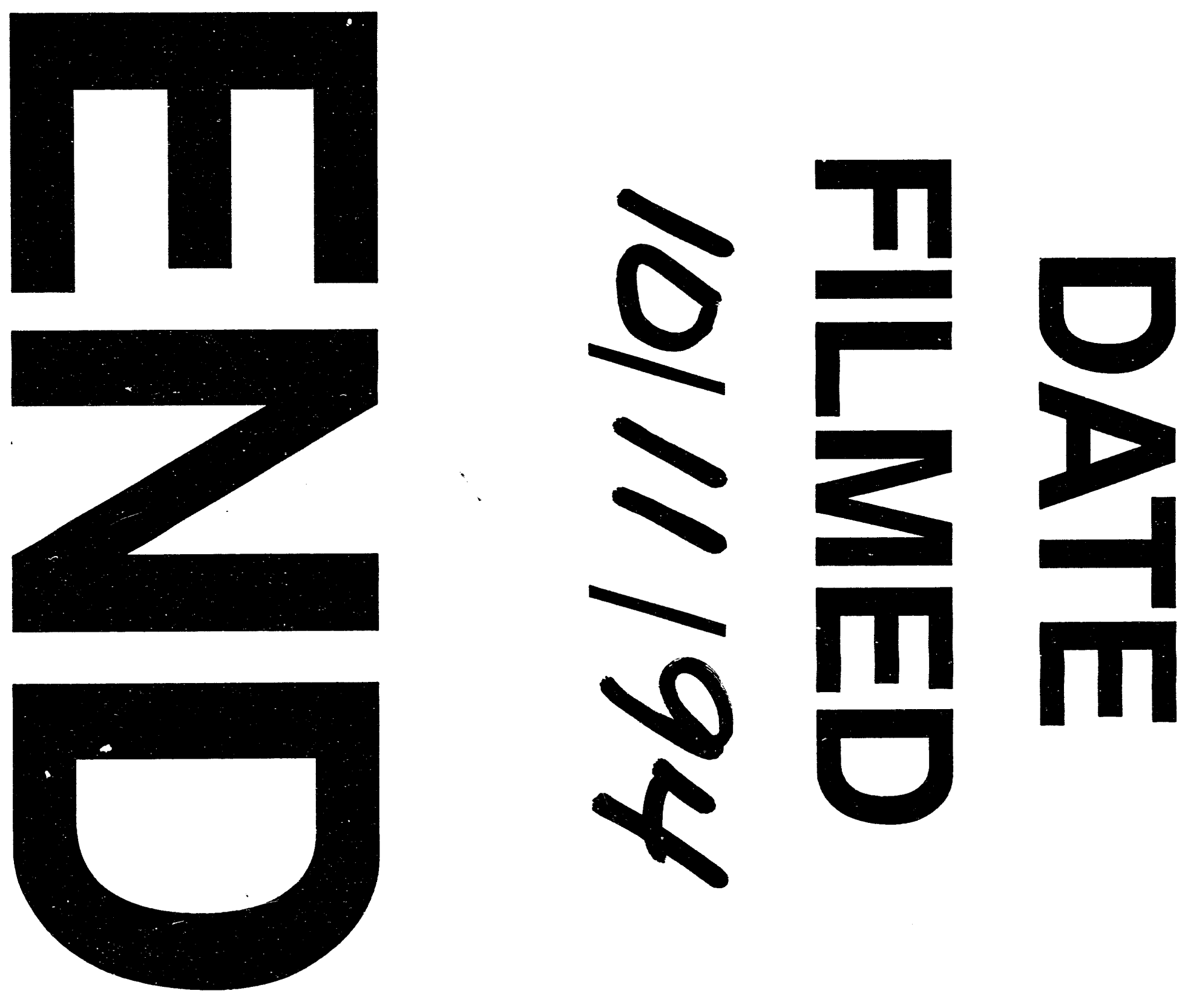


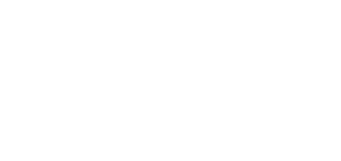

